\title{
Iterative Feedforward Tuning for Residual Vibration Reduction ${ }^{\star}$
}

\author{
Antonio Visioli * Aurelio Piazzi ** \\ * Dipartimento di Elettronica per l'Automazione, \\ University of Brescia, Italy \\ e-mail: antonio.visioli@ing.unibs.it \\ ** Dipartimento di Ingegneria dell'Informazione, \\ University of Parma, Italy \\ e-mail: aurelio.piazzi@unipr.it
}

\begin{abstract}
An iterative approach for the determination of an input-output inversion feedforward control law for residual vibration reduction is proposed in this paper. In particular, point-topoint motion planning of vibratory servosystems is considered. The method aims at estimating recursively the parameters of the system in order to determine the exact command input to be applied to the control system in order to achieve a predefined motion without oscillations. In this context, a gradient based minimisation of the integrated square error cost function is performed. Simulation results show the effectiveness of the methodology.
\end{abstract}

\section{INTRODUCTION}

It is well-known that a major source of limitation of the performance of positioning servosystems is the presence of elasticity in the transmissions that introduce vibrations. This generally implies that the working cycle time has to be increased in order for the residual oscillation to vanish after the point-to-point motion has been accomplished. As a solution to this problem, two strategies can be implemented: the closed-loop feedback control, which is based on the use of an appropriate sensor in order to measure the vibration, and the open-loop control which consists of an adequate shaping of the command input and requires the knowledge of the system model. In the first case, a relevant technique proposed recently is the wave-based control (O'Connor (2006, 2007b,a)), which is based on considering the actuator motion as launching a mechanical wave into the elastic system while, at the same time, absorbing the motion which, due to the elasticity, later returns to the actuator. The sensor is therefore placed as close as possible to the actuator, namely, as close as possible to the interface between the actuator and the flexible system.

In the context of open-loop control, the most well-known technique is the input shaping, which has been developed starting from the nineties (Singer and Seering (1990); Singhose et al. $(1994,1997))$. It consists of convolving a sequence of impulses, also known as the input shaper, with a desired system command to generate the system command that is then actually used to drive the system. The robustness of the system is addressed by using a larger number of impulses and this leads to an increasing of the motion time, because of the convolution process.

An alternative open-loop approach is that based on system inversion (Piazzi and Visioli (2000, 2001a)) which consists in first defining a desired motion of the load (without oscillations) and by subsequently determining the input

* This work was partially supported by MIUR scientific research funds. function that causes the desired motion by inverting the dynamics of the system.

In any case, it is obvious that any open-loop approach relies on the availability of an accurate model of the system. In order to estimate correctly the model parameters in the context of the inversion-based methodology, an iterative approach is presented in this paper. The proposed technique aims at estimating the parameters of the system (namely, those of the motor and of the elastic transmission) in order to minimise the integrated square error cost function, where the error considered is the difference between the obtained system transient response and the one that is desired (Piazzi and Visioli (2007)). A gradientbased minimisation is used for this purpose and the procedure exploits the use of a sensor that measures the load position. Once the optimal command input function is determined, the open-loop approach is applied.

The paper is organised as follows. In Section 2 the system model is described and the problem is formulated. The iterative methodology is described in Section 3. Simulation results are given in Section 4 and conclusions are drawn in Section 5.

\section{PROBLEM FORMULATION}

Consider a motion control system where a point-to-point motion of a load from position $y_{0}$ to position $y_{1}$ has to be performed. Without loss of generality we will assume $y_{0}=0$ hereafter. The load is connected to an actuator by means of an elastic transmission. Thus, the control scheme is the one depicted in Figure 1, where the actuator position $x$ is controlled by means of a unity-feedback ProportionalIntegral (PI) controller. If the actuator is a torque-piloted motor, it can be described by means of the following transfer function:

$$
M(s):=\frac{X(s)}{I(s)}=\frac{1 / D_{m}}{s\left(\frac{J_{m}}{D_{m}} s+1\right)}
$$


where $i$ is the input torque, $J_{m}$ is the motor inertia and $D_{m}$ is the viscous friction coefficient. The PI controller transfer function is

$$
C(s)=K_{p}\left(1+\frac{1}{T_{i} s}\right)
$$

where $K_{p}$ is the proportional gain and $T_{i}$ is the integral time constant. Note that a PI controller has been considered for simplicity but the method proposed in this paper can be applied with any controller structure.

The transmission (assuming a linear motion of the load) can be modelled as a spring in parallel with a damper and it is described by the following transfer function (Piazzi and Visioli (2000))

$$
F(s)=\frac{Y(s)}{X(s)}=\frac{c s+k}{m s^{2}+c s+k}
$$

where $m$ is the mass of the load, $k$ is the stiffness constant and $c$ the damping coefficient. Note that, in routine operation, the position $y$ of the load is not measured. The closed-loop system transfer function is therefore

$$
T(s):=\frac{X(s)}{R(s)}=\frac{C(s) M(s)}{1+C(s) M(s)}
$$

and the overall control system transfer function is

$$
H(s):=\frac{Y(s)}{R(s)}=T(s) F(s) .
$$

Now, denote as $\hat{M}\left(s ; \boldsymbol{\rho}_{M}\right)$ the estimated transfer function of the (unknown) "true" motor transfer function $M(s)$ parameterised by the parameter vector $\boldsymbol{\rho}_{M}=\left[\begin{array}{ll}J_{m} & D_{m}\end{array}\right]^{T}$ and denote as $\hat{F}\left(s ; \boldsymbol{\rho}_{F}\right)$ the estimated transfer function of the (unknown) "true" transmission transfer function $F(s)$ parameterised by the parameter vector $\boldsymbol{\rho}_{F}=\left[\begin{array}{lll}m & c k\end{array}\right]^{T}$. As a consequence, $\hat{H}(s ; \boldsymbol{\rho})$ denotes the estimated transfer function of the (unknown) "true" overall control system $H(s)$, where $\boldsymbol{\rho}=\left[\begin{array}{llll}J_{m} & D_{m} & m & c k\end{array}\right]^{T}$.

The command input function $r(\cdot)$ is determined by means of an input-output system inversion approach, which consists in defining a priori the desired load motion function $y_{d}(t)$ and then in obtaining $r(t ; \boldsymbol{\rho})$ by inverting the estimated dynamics $\hat{H}(s ; \boldsymbol{\rho})$. From a practical point of view, because the system $\hat{H}(s ; \boldsymbol{\rho})$ has a relative order equal to three, in order to obtain a continuous command input function, the desired output function has to be continuous until the third order (Piazzi and Visioli (2001b)). Further, a transition time $\tau$ has to be defined, i.e., the desired output function is defined as

$$
y_{d}(t):= \begin{cases}0 & \text { for } t<0 \\ y_{01}(t) & \text { for } 0 \leq t \leq \tau \\ y_{1} & \text { for } t>\tau\end{cases}
$$

where $y_{01}(t)$ is the desired transition function between the constant values 0 and $y_{1}$. The general procedure, which involves a numerical computation, to determine $r(\cdot)$ such that the desired output function is obtained, can be found in (Pallastrelli and Piazzi (2005)). It is worth stressing that when the selected desired output function is a polynomial function (Piazzi and Visioli (2001b)) (at least of seventh order), an analytical solution can be found (Piazzi and Visioli (2005)) and this fact can be exploited in speeding up the computational time and most of all in avoiding numerical problems.

Since the system transfer function $H(s)$ (5) has two negative zeros (one of the PI controller and one of the

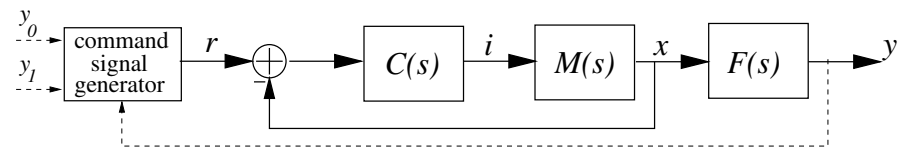

Fig. 1. The considered control system.

transmission transfer function), $r(t)$ is defined over the time interval $[0,+\infty)$ and therefore, in order to practically use it, it is necessary to truncate it. Thus, the input function exhibits a post-actuation time interval (see for example (Zou and Devasia (2004))).

In any case, since the input-output inversion procedure has necessarily to be applied to the estimated transfer function $\hat{H}(s ; \boldsymbol{\rho})$, which may encompass structured (parametric) uncertainties, the desired output function is not actually obtained in general. In other words, by applying the determined command input $r(t ; \boldsymbol{\rho})$ to the true system, the obtained system output $y(t ; \boldsymbol{\rho})$ is not equal to $y_{d}(t)$.

For this reason, an iterative strategy is proposed in order to find the optimal parameter vector defined by

$$
\boldsymbol{\rho}^{*}:=\arg \min _{\boldsymbol{\rho}} J(\boldsymbol{\rho})
$$

where $J(\boldsymbol{\rho})$ is the following integral criterion (Ho et al. (2003); Crowe and Johnson (2005)):

$$
J(\boldsymbol{\rho})=\frac{1}{2 T_{f}} \int_{0}^{T_{f}}\left(y(t ; \boldsymbol{\rho})-y_{d}(t)\right)^{2} d t
$$

where $T_{f}$ is the control interval (which might be practically selected as the sum of the transition time $\tau$ and of the post-actuation time interval).

\section{ITERATIVE FEEDFORWARD TUNING}

For the purpose of solving the optimisation problem (7), we propose to place an additional sensor in order to measure the position of the load (note that this measure is not use for feedback control) and then to employ a gradientbased minimisation, namely, the following expression can be used after the $i$ th iteration:

$$
\boldsymbol{\rho}(i+1)=\boldsymbol{\rho}(i)-\gamma_{i} \mathbf{R}_{i}^{-1} \frac{\partial J}{\partial \boldsymbol{\rho}}(\boldsymbol{\rho}(i))
$$

where $\gamma_{i}$ is a positive real scalar that determines the step size and $R$ is some appropriate positive definite matrix.

\subsection{Generating the cost function gradient}

The computation of the cost function gradient is therefore necessary in order to apply the gradient search (9). From (8) it follows that

$$
\frac{\partial J}{\partial \boldsymbol{\rho}}(\boldsymbol{\rho}(i))=\frac{1}{T_{f}} \int_{0}^{T_{f}} e(t ; \boldsymbol{\rho}(i)) \frac{\partial e(t ; \boldsymbol{\rho}(i))}{\partial \boldsymbol{\rho}} d t
$$

where

$$
e(t ; \boldsymbol{\rho})=y(t ; \boldsymbol{\rho})-y_{d}(t) .
$$

It can be noted that $e(t ; \boldsymbol{\rho}(i))$ is immediately available after an experiment has been performed. In order to determine an expression of the first derivative of the error signal, since an analytical expression cannot be derived in general, it is convenient to consider the Laplace transform of the functions considered, namely,

$$
E(s ; \boldsymbol{\rho})=Y(s ; \boldsymbol{\rho})-Y_{d}(s)=H(s) R(s ; \boldsymbol{\rho})-Y_{d}(s) .
$$


By considering that $R(s ; \boldsymbol{\rho})$ has been obtained by inverting the system $\hat{H}(s ; \boldsymbol{\rho})$, we can write

$$
\begin{aligned}
R(s ; \boldsymbol{\rho})= & \hat{H}^{-1}(s ; \boldsymbol{\rho}) Y_{d}(s)= \\
& {\left[\frac{1+C(s) \hat{M}\left(s ; \boldsymbol{\rho}_{M}\right)}{C(s) \hat{M}\left(s ; \boldsymbol{\rho}_{M}\right) \hat{F}\left(s ; \boldsymbol{\rho}_{F}\right)}\right] Y_{d}(s) }
\end{aligned}
$$

and, hence, we obtain, after trivial calculations:

$$
\begin{aligned}
\frac{\mathrm{d} E(s ; \boldsymbol{\rho})}{\mathrm{d} \boldsymbol{\rho}}= & \frac{H(s ; \boldsymbol{\rho}) Y_{d}(s)}{C(s) \hat{M}^{2}\left(s ; \boldsymbol{\rho}_{M}\right) \hat{F}^{2}\left(s ; \boldsymbol{\rho}_{F}\right)} . \\
& \left(-\hat{F}\left(s ; \boldsymbol{\rho}_{F}\right) \frac{\partial \hat{M}\left(s ; \boldsymbol{\rho}_{M}\right)}{\partial \boldsymbol{\rho}}\right. \\
& -\hat{M}\left(s ; \boldsymbol{\rho}_{M}\right) \frac{\partial \hat{F}\left(s ; \boldsymbol{\rho}_{F}\right)}{\partial \boldsymbol{\rho}} \\
& \left.-C(s) \hat{M}^{2}\left(s ; \boldsymbol{\rho}_{M}\right) \frac{\partial \hat{F}\left(s ; \boldsymbol{\rho}_{F}\right)}{\partial \boldsymbol{\rho}}\right) .
\end{aligned}
$$

At this point it is worth considering the three addenda (14)-(16) separately. Indeed, (14) can be rewritten as

$$
\begin{aligned}
& -H(s ; \boldsymbol{\rho}) Y_{d}(s) \frac{\partial \hat{M}\left(s ; \boldsymbol{\rho}_{M}\right)}{\partial \boldsymbol{\rho}} \frac{1}{C(s) \hat{M}^{2}\left(s ; \boldsymbol{\rho}_{M}\right) \hat{F}\left(s ; \boldsymbol{\rho}_{F}\right)} \\
& \cdot \frac{1+C(s) \hat{M}\left(s ; \boldsymbol{\rho}_{M}\right)}{1+C(s) \hat{M}\left(s ; \boldsymbol{\rho}_{M}\right)}= \\
& =-\frac{\partial \hat{M}\left(s ; \boldsymbol{\rho}_{M}\right)}{\partial \boldsymbol{\rho}} \frac{1}{1+C(s) \hat{M}\left(s ; \boldsymbol{\rho}_{M}\right)} \frac{1}{\hat{M}\left(s ; \boldsymbol{\rho}_{M}\right)} Y(s ; \boldsymbol{\rho}) \\
& =: P_{1}\left(s ; \boldsymbol{\rho}_{M}\right) Y(s ; \boldsymbol{\rho})
\end{aligned}
$$

The, (15) can be rewritten as

$$
\begin{aligned}
& -H(s ; \boldsymbol{\rho}) Y_{d}(s) \frac{\partial \hat{F}\left(s ; \boldsymbol{\rho}_{F}\right)}{\partial \boldsymbol{\rho}} \frac{1}{C(s) \hat{M}\left(s ; \boldsymbol{\rho}_{M}\right) \hat{F}^{2}\left(s ; \boldsymbol{\rho}_{F}\right)} \\
& \cdot \frac{1+C(s) \hat{M}\left(s ; \boldsymbol{\rho}_{M}\right)}{1+C(s) \hat{M}\left(s ; \boldsymbol{\rho}_{M}\right)}= \\
& =-\frac{\partial \hat{F}\left(s ; \boldsymbol{\rho}_{F}\right)}{\partial \boldsymbol{\rho}} \frac{1}{1+C(s) \hat{M}\left(s ; \boldsymbol{\rho}_{M}\right)} \frac{1}{\hat{F}\left(s ; \boldsymbol{\rho}_{F}\right)} Y(s ; \boldsymbol{\rho}) \\
& =: P_{2}(s ; \boldsymbol{\rho}) Y(s ; \boldsymbol{\rho})
\end{aligned}
$$

Finally, (16) can be rewritten as

$$
\begin{aligned}
& -H(s ; \boldsymbol{\rho}) Y_{d}(s) \frac{\partial \hat{F}\left(s ; \boldsymbol{\rho}_{F}\right)}{\partial \boldsymbol{\rho}} \frac{1}{\hat{F}^{2}\left(s ; \boldsymbol{\rho}_{F}\right)} \\
& \cdot \frac{1+C(s) \hat{M}\left(s ; \boldsymbol{\rho}_{M}\right)}{1+C(s) \hat{M}\left(s ; \boldsymbol{\rho}_{M}\right)} \cdot \frac{C(s) \hat{M}\left(s ; \boldsymbol{\rho}_{M}\right)}{C(s) \hat{M}\left(s ; \boldsymbol{\rho}_{M}\right)}= \\
& =-\frac{\partial \hat{F}\left(s ; \boldsymbol{\rho}_{F}\right)}{\partial \boldsymbol{\rho}} \frac{1}{\hat{F}\left(s ; \boldsymbol{\rho}_{F}\right)} \hat{T}\left(s ; \boldsymbol{\rho}_{M}\right) Y(s ; \boldsymbol{\rho}) \\
& =: P_{3}(s ; \boldsymbol{\rho}) Y(s ; \boldsymbol{\rho}) .
\end{aligned}
$$

It turns out that, by considering the corresponding timedomain signals, the term $\partial e(t ; \boldsymbol{\rho}) / \partial \boldsymbol{\rho}$ can be computed by determining the response of the systems $P_{1}\left(s ; \boldsymbol{\rho}_{M}\right)$, $P_{2}(s ; \boldsymbol{\rho})$, and $P_{3}(s ; \boldsymbol{\rho})$ to the obtained output $y(t ; \boldsymbol{\rho})$ and by adding the results.

\subsection{Algorithm}

Based on the above considerations, given the estimated system transfer functions $\hat{M}\left(s ; \boldsymbol{\rho}_{M}\right)$ and $\hat{F}\left(s ; \boldsymbol{\rho}_{F}\right)$, a con- troller transfer function $C(s)$ and a desired output function $y_{d}(t)$, the overall Iterative FeedForward Tuning (IFFT) algorithm for residual vibration reduction can be outlined as follows.

\section{IFFT algorithm}

(1) Choose a parameter vector $\rho$

(2) Determine the command input function $r(t ; \boldsymbol{\rho})$ by applying an input-output inversion procedure to the closed-loop system $\hat{H}(s ; \boldsymbol{\rho})$ with output function $y_{d}(t)$.

(3) Run a closed-loop system experiment with command input $r(t ; \boldsymbol{\rho})$ and measure the load position $y(t ; \boldsymbol{\rho})$.

(4) Record the system output $y(t ; \boldsymbol{\rho})$ and determine the error signal $e(t ; \boldsymbol{\rho})=y(t ; \boldsymbol{\rho})-y_{d}(t)$.

(5) Determine $\partial e(t ; \boldsymbol{\rho}) / \partial \boldsymbol{\rho}$ as the response of the system $P_{1}\left(s ; \boldsymbol{\rho}_{M}\right)+P_{2}(s ; \boldsymbol{\rho})+P_{3}(s ; \boldsymbol{\rho})($ see $(17)-(19))$ to the signal $y(t ; \boldsymbol{\rho})$.

(6) Calculate the cost function gradient (10).

(7) If $\|\partial J(\boldsymbol{\rho}(i)) / \partial \boldsymbol{\rho}\|>\varepsilon$ then update the parameter vector $\boldsymbol{\rho}$ by applying formula (9) and go to 2 .

(8) Apply the determined command input $r\left(t ; \boldsymbol{\rho}^{*}\right)$ repeatedly in the routine system operations (the measure of $y(t)$ is no more necessary).

Note that, although the algorithm converges to the set of stationary points of the criterion (8) (see Section 4), the termination condition at step 7 is employed in order to avoid a infinite number of iterations. In this context the user-chosen parameter $\varepsilon$ allows to handle the tradeoff between the number of iterations and the achieved performance.

\section{PRACTICAL ISSUES}

A few practical issues have to be addressed for an effective implementation of the procedure described in Section 3. First, it has to be stressed that, since all the adopted signals are bounded, the convergence properties of the algorithm can be derived by following an analysis analogous to the one of the Iterative Feedback Tuning methodology (Hjalmarsson et al. (1998); Hjalmarsson (2002)). Further, the considerations made in (Hjalmarsson et al. (1998); Hjalmarsson (2002)) regarding the choice of the sequence of matrices $\mathbf{R}_{i}$ in (9) apply also for the IFFT technique. It is also worth stressing at this point that the presence of measurement noise (which has not been taken into account explicitly in the previous analysis) can be addressed by an appropriate filtering of the data. Note that a standard offline filtering technique can be employed.

The IFFT algorithm has been devised on the basis that only structured uncertainties are taken into account explicitly. In any case it has to be considered that the presence of the feedback controller reduces the effects of the motor unstructured uncertainties at low frequencies where the determined command input has its frequency content (Piazzi and Visioli (2006b)). In this context a role is played by the transition time $\tau$ (see (6)) that allows also to handle the actuator constraints (and therefore to avoid detrimental nonlinear effects). This is also the reason for which it is not necessary to include a term related to the control effort in the considered cost function (8).

Finally, note that, in order to provide a useful tool for 


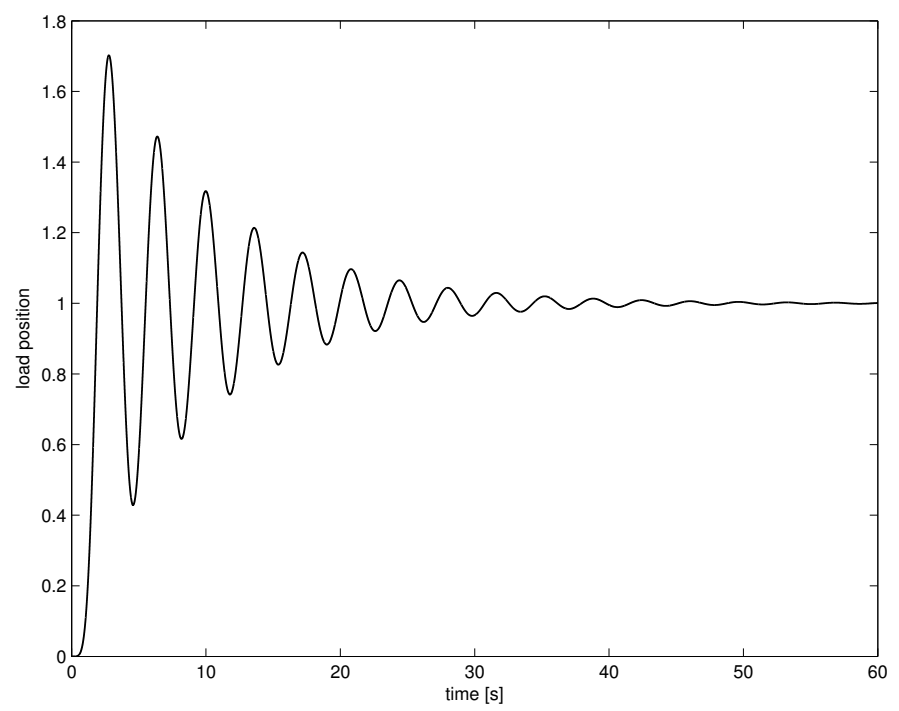

Fig. 2. The load motion obtained by using the desired output function as a command input.

the application of a the input-output inversion procedure (step 2 of the algorithm), a Matlab toolbox has been implemented (Piazzi and Visioli (2006a)).

\section{SIMULATION RESULTS}

\subsection{Example 1}

As a first illustrative example we considered the system shown in Figure 1 with $J_{m}=0.00011, D_{m}=0.0034$, $m=1, c=0.22$ and $k=3.05$. As a desired output function (see $(6)$ ), for a transition from 0 to $y_{1}=1$, a seventh-order polynomial has been selected, namely,

$$
y_{01}=-20 \frac{t^{7}}{\tau^{7}}+70 \frac{t^{6}}{\tau^{6}}-84 \frac{t^{5}}{\tau^{5}}+35 \frac{t^{4}}{\tau^{4}}
$$

where $\tau=2$. In order to evaluate the significance of the elasticity, the load positon obtained when the desired (smooth) output function is employed as a command input is shown in Figure 2, where the residual vibration emerges clearly. Then, the iterative procedure has been applied, starting from an initial estimate of the parameters $\rho(1)=$

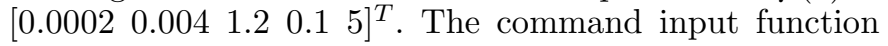
and the load motion obtained at the first iteration are plotted in Figures 3 and 4 respectively. By setting for any $i \gamma_{i}=10^{-3}$ and

$$
\mathbf{R}_{i}^{-1}=\operatorname{diag}\left(10^{-3}, 10^{-3}, 1,1,10\right)
$$

at the tenth iteration the command input and the load motion shown in Figures 5 and 6 respectively has been obtained. The improvement is evident and it is confirmed by the values of the cost function $J(\boldsymbol{\rho})$ at the different iterations shown in Figures 7. The parameters vector at the tenth iteration is $\boldsymbol{\rho}(10)=$ $\left[\begin{array}{lllll}0.00028 & 0.004 & 1.2 & 0.08 & 4.99\end{array}\right]^{T}$ demonstrating that a small change in the parameters might have a significant effect on the performance achieved.

\subsection{Example 2}

As a second example, we consider the same system of example 1, but in this case the initial parameters

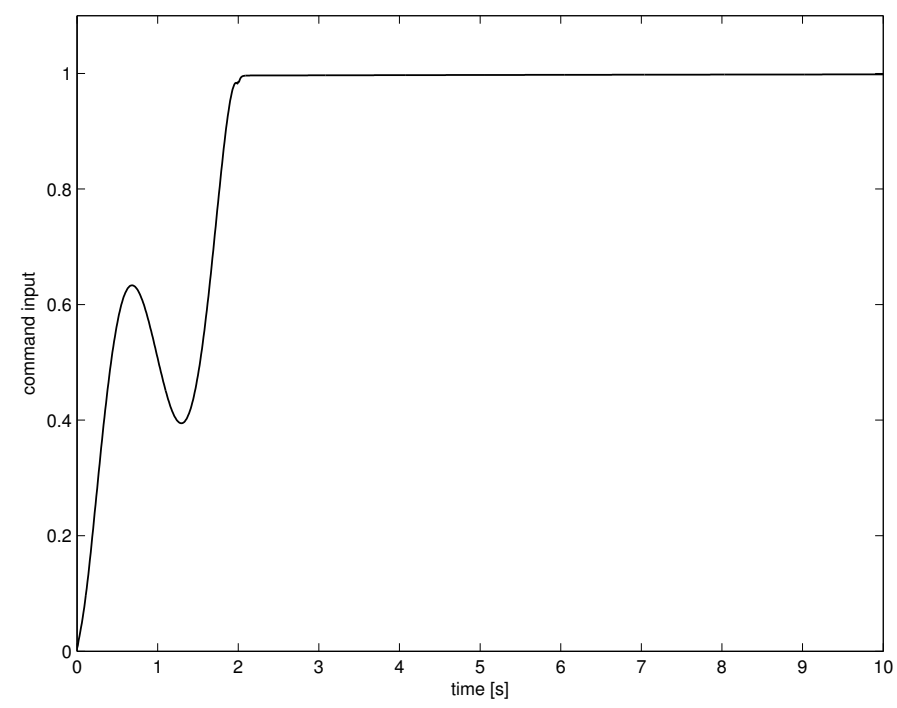

Fig. 3. Command input at the first iteration - example 1.

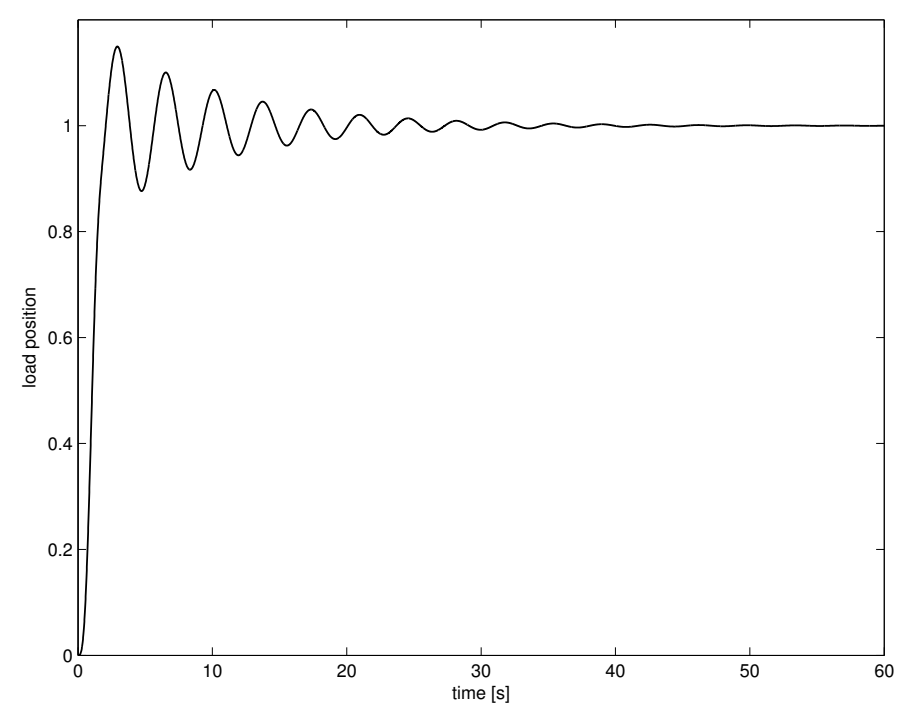

Fig. 4. Load motion at the first iteration for example 1.

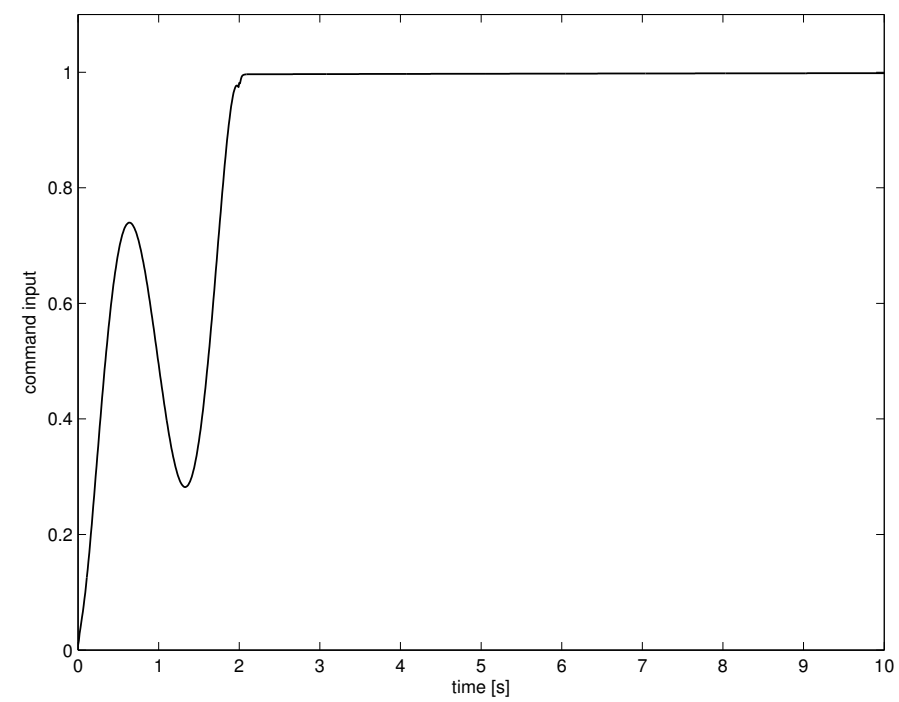

Fig. 5. Command input at the tenth iteration - example 1. 


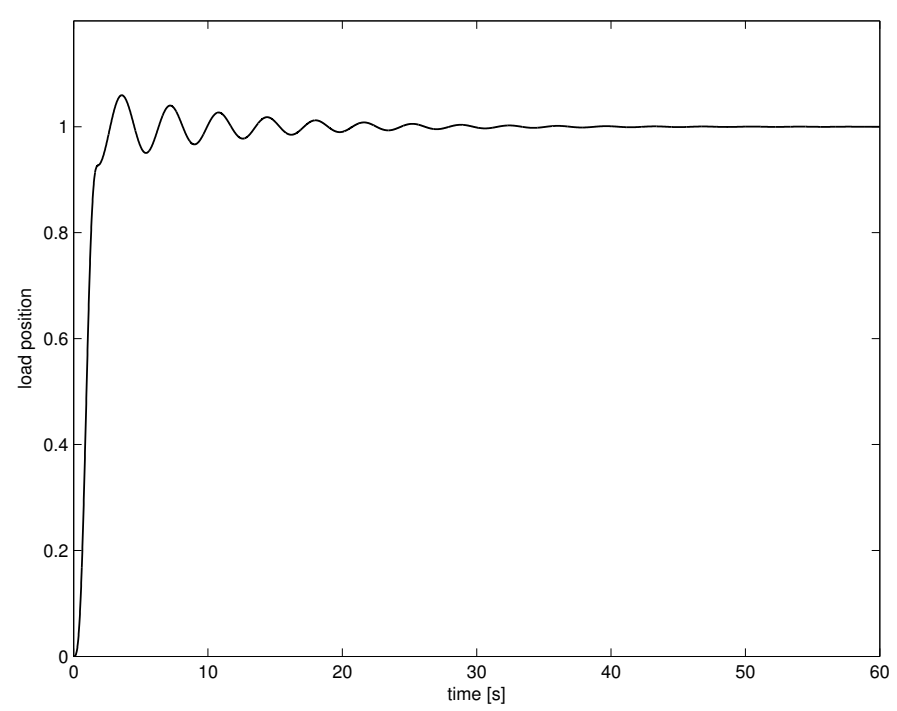

Fig. 6. Load motion at the tenth iteration - example 1.

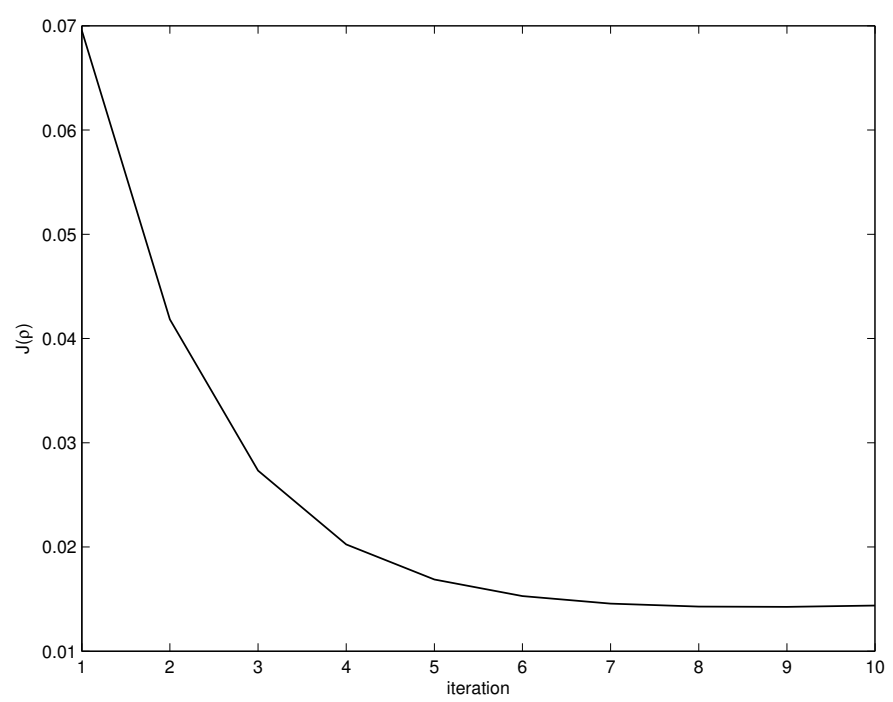

Fig. 7. Cost function - example 1.

vector is $\boldsymbol{\rho}(1)=\left[\begin{array}{lllll}0.0001 & 0.002 & 0.9 & 0.1 & 5\end{array}\right]^{T}$. Results are shown in Figures 8-12 and in this case $\boldsymbol{\rho}(10)=$ $\left[\begin{array}{lllll}0.00089 & 0.0016 & 0.87 & 0.09 & 5.05\end{array}\right]^{T}$. It turns out that also in this case the IFFT procedure is capable to reduce the residual vibration significantly.

\section{CONCLUSIONS}

A methodology for the residual vibration reduction in vibratory servosystems has been presented in this paper. In particular, a suitable inversion-based feedforward control law is determined by means of an iterative gradient-based optimisation procedure. Although the devised tecnique yields in general to a local optimum, it appears that just a few iteration are necessary to improve significantly the performance of the servosystem.

\section{REFERENCES}

J. Crowe and M. A. Johnson. On-line model-free methods. In PID Control - New Identification and Design Methods

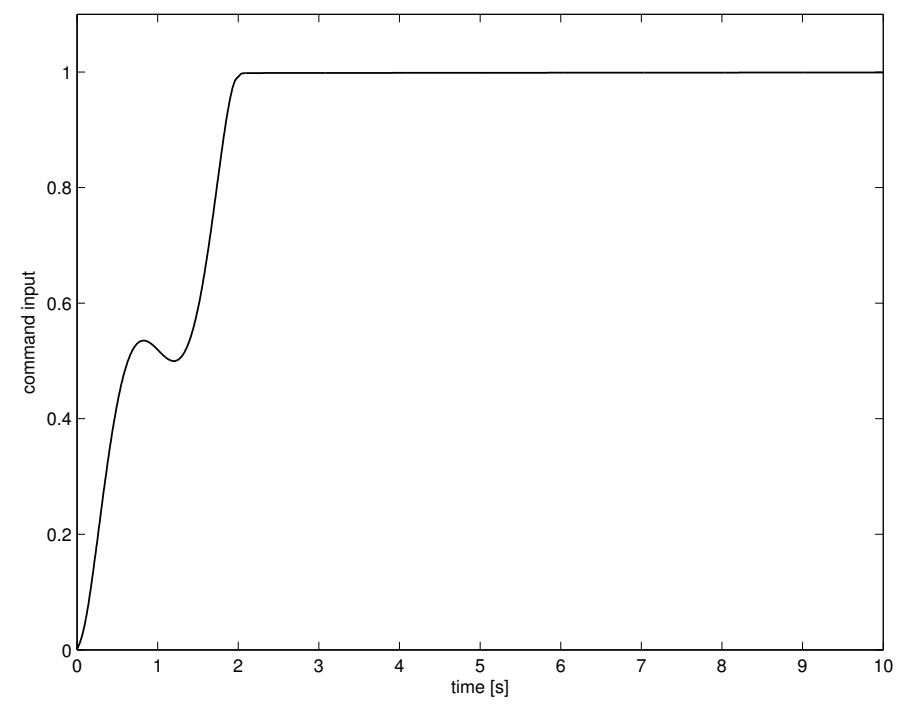

Fig. 8. Command input at the first iteration - example 2.

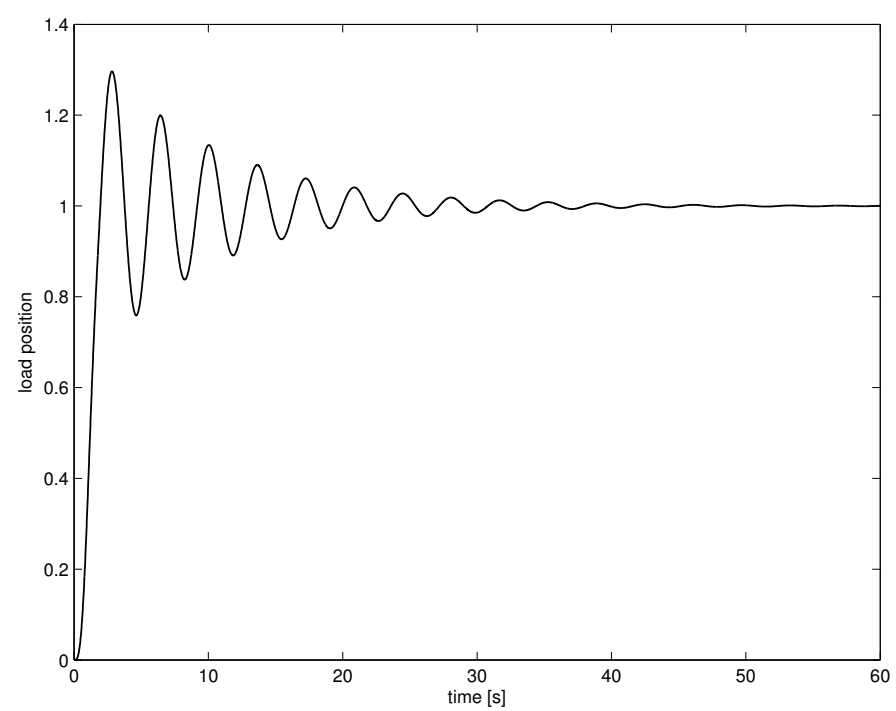

Fig. 9. Load motion at the first iteration - example 2.

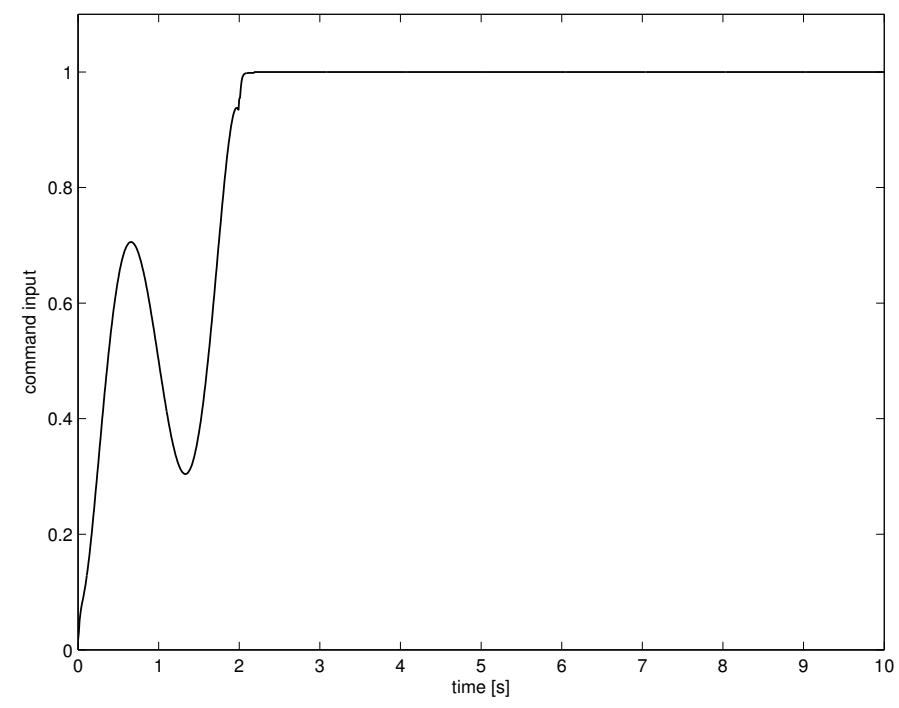

Fig. 10. Command input at the tenth iteration - example 1. 


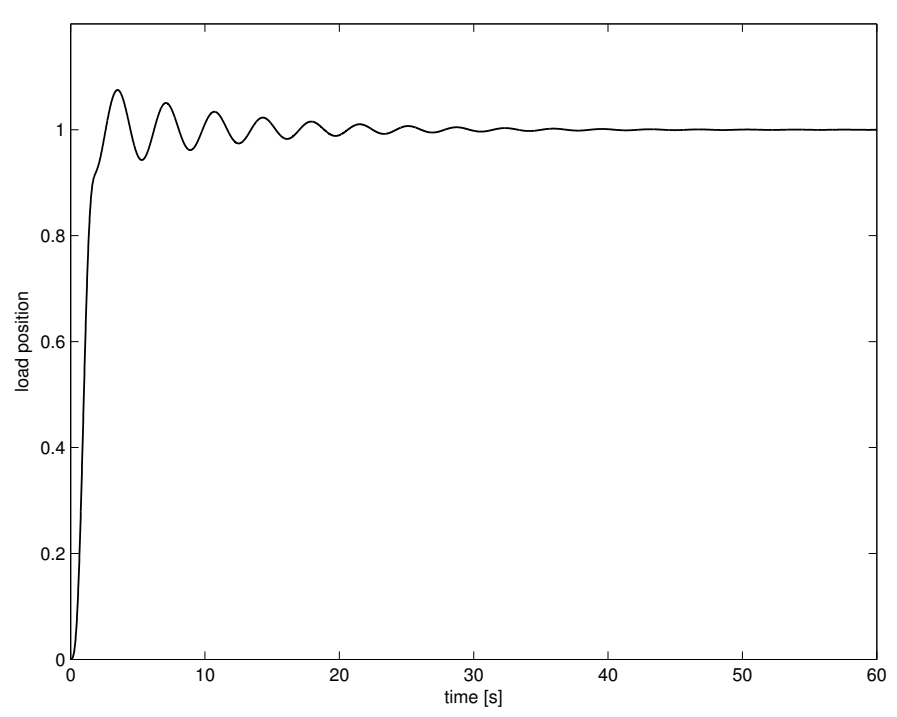

Fig. 11. Load motion at the tenth iteration - example 2.

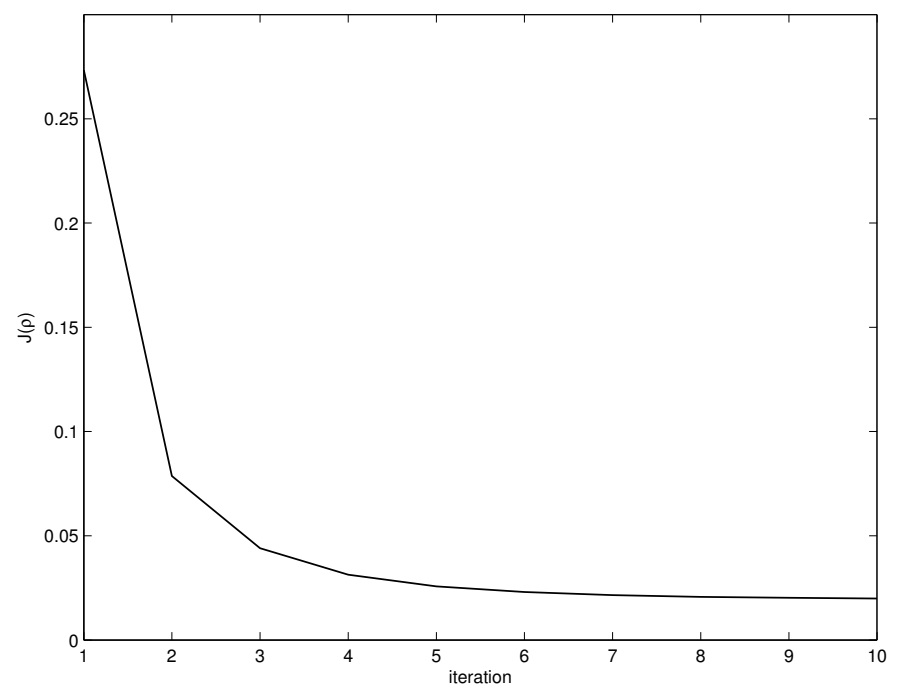

Fig. 12. Cost function - example 2.

(M. A. Johnson and M. M. Moradi eds.), London, UK, 2005. Springer.

H. Hjalmarsson. Iterative feedback tuning - an overview. International Journal of Adaptive Control and Signal Processing, 16:373-395, 2002.

H. Hjalmarsson, M. Gevers, S. Gunnarsson, and O. Lequin. Iterative feedback tuning: theory and applications. IEEE Control Systems Magazine, 18:26-41, 1998.

W. K. Ho, Y. Hong, A. Hansson, H. Hjalmarsson, and J. W. Deng. Relay auto-tuning of pid controllers using iterative feedback tuning. Automatica, 39:149157, 2003.

W. J. O'Connor. Control of flexible mechanical systems: wave-based techniques. In Proceedings American Control Conference, pages 4192-4202, New York (NY), 2007a.

W. J. O'Connor. Wave-echo control of lumped flexible systems. Journal of Sound and Vibration, 298:10011018, 2006.

W. J. O'Connor. Wave-based analysis and control of lumped-modelled flexible robots. IEEE Transactions on
Robotics, 2007b.

D. Pallastrelli and A. Piazzi. Stable dynamic inversion of nonminimum-phase scalar linear systems. In Preprints of the 16th IFAC World Congress on Automatic Control, Prague (CZ), 2005.

A. Piazzi and A. Visioli. An iterative approach for noncausal feedforward tuning. In Proceedings American Control Conference, pages 1251-1256, New York, NY, 2007.

A. Piazzi and A. Visioli. A toolbox for input-output system inversion. In Preprints of the 7th IFAC Symposium on Advances in Control Education, Madrid (E), 2006a.

A. Piazzi and A. Visioli. Point-to-point motion planning for servosystems with elastic transmission via optimal dynamic inversion. ASME Journal of Dynamic Systems, Measurement, and Control, 123:733-736, 2001a.

A. Piazzi and A. Visioli. Optimal noncausal set-point regulation of scalar systems. Automatica, 37:121-127, 2001b.

A. Piazzi and A. Visioli. Using stable input-output inversion for minimum-time feedforward constrained regulation of scalar systems. Automatica, 41:305-313, 2005.

A. Piazzi and A. Visioli. Minimum-time system-inversionbased motion planning for residual vibration reduction. IEEE/ASME Transactions on Mechatronics, 5(1):12$22,2000$.

A. Piazzi and A. Visioli. Combining $h_{\infty}$ control and dynamic inversion for robust constrained set-point regulation. In Preprints IFAC Workshop on Control Applications of Optimisation, pages 209-214, Paris-Cachan (F), 2006b.

N. C. Singer and W. P. Seering. Preshaping command inputs to reduce system vibration. ASME Journal of Dynamic Systems, Measurement, and Control, 112:7682, 1990 .

W. Singhose, W. Seering, and N. Singer. Residual vibration reduction using vector diagrams to generate shaped inputs. ASME Journal of Mechanical Design, 116:654$659,1994$.

W. E. Singhose, L. J. Porter, T. D. Tuttle, and N. C. Singer. Vibration reduction using multi-hump input shapers. ASME Journal of Dynamic Systems, Measurement, and Control, 119:320-326, 1997.

Q. Zou and S. Devasia. Preview-based inversion of nonlinear nonminimum-phase systems: VTOL example. In Proceedings IEEE International Conference on Decision and Control, pages 4350-4356, Paradise Island, The Bahamas, 2004. 